JICA

27,1

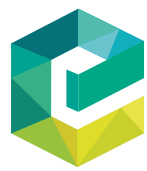

Journal of Integrated Care Vol. 27 No. 1, 2019 pp. $2-4$

(C) Emerald Publishing Limite 1476-9018

DOI 10.1108/JICA-02-2019-066

\title{
Editorial
}

\section{How organisation theory may help us understand integrated care}

Integrated care has come a long way. From its first tentative steps in the 1990s, it has now developed into a mature field of scientific inquiry with its own associations and think tanks, special interest groups and dedicated policies focussing on designing and implementing integrated care solutions within and across professional sectors. It has also spawned an enormous amount of conceptual and empirical research.

Despite all the progress, there remains a lingering question at the heart of the discipline. Why do organisations do integrated care? This is not to question personal motives for integrated care. We know that integrated care solutions deliver better conditions for professionals, which ultimately may lead to improved patient care. A recent systematic review indicated that integrated care models demonstrated positive impacts in at least two of the triple aims, access and patient care quality (Baxter et al., 2018).

But why would organisations want to embark on integration programmes as opposed to any other transformational programme? At a first glance, the answer to the question appears to be obvious. Organisations integrate because they have to. As often noted, most integrated care projects are instigated through top-down policy initiatives. Genuine bottom up integration is rare. And the preference for a top-down approach has consequences for the organisations mandated to integrate. Where the options of avoiding a policy are few, existing asymmetries of power and resources are often reinforced and cemented among participating services rather than mitigated.

On the other hand, the motivational glue that holds integrated care policies together is of course the desire to improve patient care. I have previously expressed some scepticism that this is the most powerful or, indeed even the most honest description of staff motivations when implementing integrated care (Kaehne, 2018). Two arguments counsel against blind faith in this often professed professional impulse; first, the relative paucity of evidence of integrated care on patient care quality; and second, the fact that integrated care solutions are just one among other options to improve patient care. In fact, the complexity of care integration is such that I doubt it would be the first choice of service managers and their staff when faced with the need to change. Integrated care is notoriously difficult to design, and its impact is difficult to predict. In addition, where organisational change reduces certainties, risks to core operational tasks are increasing. So why would anyone embark on a risky strategy if you could try out others, less risky ones first?

While this argument reveals the policy pressures that often underpin integration programmes imposed by central or local governments, there may be other perspectives that throw light on some issues that tempt organisations onto a path towards integration. Using organisation theory, I will detail a few below.

Despite all the useful conceptual research in the integration field, applications of organisation theory are relatively rare in health studies. This may be due to a reluctance to see parallels between commercial activity and healthcare. Yet, where organisation theory says something about how organisations change and why, it may give us additional insights into how healthcare organisations behave in the face of imposed change. While granting substantial differences between commercial organisations and healthcare services, there may still be some intriguing similarities that tell us more about integrated care programmes.

Contingency theory is probably the most obvious recent emanation of organisation theory to be applied in the healthcare context (McKinley and Mone, 2003). In effect, it says 
that organisations operate under conditions of uncertainty which they are trying to reduce through adaptation. Where variables of contingency interject into an equilibrium (defined as market balance for commercial organisations), they try to change to make themselves "fit" to new circumstances. Recognising the terminological ambiguity of the construct "fit", we can still use contingency theory as a heuristic device to structure the processes of change in a healthcare organisation as and when integration occurs. Integrated care projects are responses to variables that shift the policy framework within which all services operate. They are responses to contingent factors interjected into the system from outside, typically policies from the centre of political gravity.

A key characteristic of contingency theory in the commercial sector is that organisations react to external stimuli in such a way that protects their existence and maximises their profits (or utility). The key insight from looking at health services through the prism of contingency theory in moments of care integration is that we assume patient care to be the core utility of health providers and hence that their adaption to the contingency variable (the integration demand) would automatically lead to an improvement of care quality as organisations maximise their core business product. Yet, as Lipsky (1980) observed, the main utility to be pursued by healthcare organisations is their internal integrity and accountability to the various targets and objectives set by government and professional guidelines, not (necessarily) patient care.

So where care integration policies interject into business as usual in healthcare organisations, the main trajectory of the adaption process is more likely to be aimed at securing the integrity of the organisation and to re-establish effective rules of engagement between staff to create the equilibrium temporarily lost, rather than improve patient care. Of course, both are not mutually exclusive but neither are they necessarily correlated. More likely, there is an incidental relationship between the two where any organisational changes bring about some care quality improvements by virtue of disrupting some ineffective or detrimental practices as well as some effective ones.

The second useful offering from organisation theory may be resource dependency theory. Again, we would be after its heuristic function here rather than use it as a fully fledged explanatory theory. Strikingly, resource dependence theory does tell us something important about a key area of integration, interorganisational relations. Its core thesis is that where there is interdependence between organisations on issues such as supply of goods or services, organisations gravitate towards vertical or horizontal integration to reduce costs and increase efficiencies. A central theme in this re-organisation is the (re-)defining of boundaries, including or excluding others from access to resources. Like contingency theory, resource dependency theory rests on the assumption of managerial rationality as a core characteristic of operations. It also says something about the motivations and strategies to be employed to ensure the integrity and sustainability of the organisation. To survive, organisations collaborate or merge with others, building alliances, partnerships or "trusts" defined by mutual interest and shared objectives.

The critical aspect of resource dependency theory for healthcare, however, lies in the way in which it conceptualises the path to integration (or dis-integration). The dependence of an organisation on another organisation to deal with contingencies is a crucial deciding variable as to how integration (i.e. defining organisational boundaries) plays out for everyone involved. Those who adapt well to contingent external factors accrue coping capabilities that are in turn translated into power to determine where the organisational boundaries fall.

The resemblances with integration processes across the health and social care sectors are striking. If we understand the term resource broadly, in terms of status, access to financial resources or discursive hegemony in public debate, we can see how healthcare organisations accrue substantially more adaptive capacities than other care organisations. The result is 
integration on the basis of a widening influence of health systems, encroaching into the delivery domains of social care and preventative services, often on the basis of the medical model. Examples would be multi-speciality providers merging with, or expanding into, community provision, or large hospital trusts arrogating services to themselves that hitherto were separately provided by community care providers.

Resource dependency theory reminds us that policy is first and foremost the injection of uncertainty into a system by changing the power distribution between organisations. In this sense, resource dependency theory tells us something about the likely dynamics of how integration plays out in contexts marked by significant power, status and resource asymmetries. The intriguing insight is, once again, that patient care figures surprisingly little in this integration scenario. Whichever organisation copes better with the uncertainties of the policy landscape will determine the shape of the integrated care eventually delivered on the ground.

Where organisational capacity to develop effective coping strategies with contingencies and resource dependencies determine the setup of care delivery, patient preferences will struggle to make themselves felt. Surely, they are important to healthcare organisations as a parameter for measuring service outcomes, but given infinite demand, the main benchmark of managerial strategy is creating certainties in the system, rather than the quality of patient care.

Admittedly, these are not empirically evidenced insights into how integration happens or why. But they can shape our conceptual map of integrated care programmes. And, crucially, they should inform our expectations about care integration, plausibly attenuating our hopes of radical patient care improvements through organisational change.

Axel Kaehne

\section{References}

Baxter, S., Johnson, M., Chambers, D., Sutton, A., Goyder, E. and Booth, A. (2018), "The effects of integrated care: a systematic review of UK and international evidence", BMC Health Services Research, Vol. 18 No. 1, p. 350, available at: http://doi.org/10.1186/s12913-018-3161-3

Kaehne, A. (2018), “The tacit assumptions of care integration”, Journal of Integrated Care, Vol. 26 No. 4, pp. 254-256, available at: http://doi.org/10.1108/JICA-10-2018-057

Lipsky, M. (1980), Street-Level Bureaucracy. The Dilemmas of the Individual in Public Services, Russell Sage Foundation, New York, NY.

McKinley, W. and Mone, M.A. (2003), "Micro and macro perspectives in organization theory: a tale of incommensurability", in Tsoukas, H. and Knudsen, C. (Eds), The Oxford Handbook of Organization Theory, Oxford University Press, Oxford, pp. 345-372. 